\title{
Brief
}

\section{Transaortic injection technique in fluorescence imaging: Novel intraoperative assessment of anastomosis in off-pump coronary artery bypass grafting}

Tamotsu Yasuda, MD, PhD, Go Watanabe, MD, PhD, and Shigeyuki Tomita, MD, PhD, Kanazawa, Japan

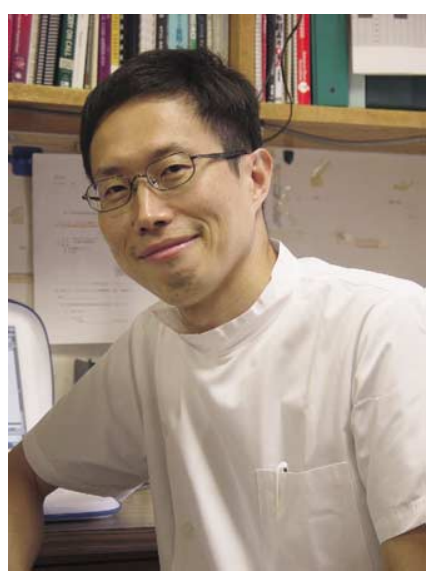

Dr Yasuda
From the Department of General and Cardiothoracic Surgery, Kanazawa University School of Medicine, Kanazawa, Japan.

Received for publication Dec 2, 2004; accepted for publication Dec 10, 2004.

Address for reprints: Tamotsu Yasuda, $\mathrm{MD}, \mathrm{PhD}$, Department of General and Cardiothoracic Surgery, Kanazawa University School of Medicine, Takaramachi 13-1, Kanazawa, Ishikawa 920-8641, Japan (Email: tamotsuyasuda@aol.com).

J Thorac Cardiovasc Surg 2005;130:560-1

$0022-5223 / \$ 30.00$

Copyright $\odot 2005$ by The American Association for Thoracic Surgery

doi:10.1016/j.jtcvs.2004.12.014

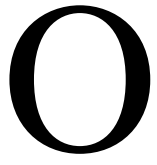

ff-pump coronary artery bypass grafting (OPCAB) has been gaining in popularity in recent years. OPCAB might decrease the mortality and morbidity related to cardiopulmonary bypass in cardiac surgery. Although some devices, including heart stabilizers or heart positioners, have evolved in recent years, one of the concerns regarding OPCAB is the quality of anastomoses. ${ }^{1}$

Recently, Taggart and colleagues ${ }^{2}$ reported that intraoperative fluorescence imaging of coronary grafts is a simple, safe, noninvasive, and reproducible technique in $\mathrm{OPCAB},{ }^{2}$ and it can allow surgeons to make immediate surgical revision if necessary. However, some surgeons noticed that it is difficult to assess the quality of an anastomosis with this technique if the native coronary artery is not so narrowed. In that case, the native coronary artery can be seen first, and then the left internal thoracic artery (LITA) is enhanced afterward because dye is injected into the central venous system.

In this report we describe a new and simple injection technique using indocyanine green (ICG) and the efficacy of the rapid imaging technique for intraoperative assessment of a LITA graft in OPCAB.

\section{Clinical Summary}

Intraoperative fluorescence imaging was performed in 10 patients who underwent OPCAB at Kanazawa University Hospital in 2004. The LITA was harvested in a semiskeletonized fashion, and after anastomosis onto the left anterior descending artery (LAD), the LITA was completely skeletonized from the anastomotic site to $5 \mathrm{~cm}$ upstream of that site. Fluorescence imaging of the LITA-LAD graft with ICG was performed after all the target coronary arteries were anastomosed. Epiaortic echocardiography was performed in all cases to examine the aortic wall. Two milliliters of ICG was injected directly into the ascending aorta just below the brachiocephalic artery with a 23-gauge fine needle, and the image was recorded immediately after injection. It usually took only 2 or 3 seconds to inject ICG, and an additional stitch for hemostasis was not needed. Unlike injection into the central venous system (Figure 1), native coronary arteries were not enhanced while recording, and the skeletonized distal LITA was easily recognized. We always obtained clear images of the LITA-LAD anastomosis, and it might be equivalent to the contrast enhancement in radiography with a catheter (Figure 2). Moreover, it was also easy to assess the patency of the right internal thoracic artery or the gastroepiploic artery.

\section{Discussion}

There are several ways to assess graft patency intraoperatively, including echocardiography, ${ }^{3}$ thermal angiography, ${ }^{4}$ Doppler ultrasonography, and angiography. ${ }^{5}$ However, all of these have limitations in terms of quality assessment of anastomosis, invasiveness, and x-ray exposure. Needless to say, whether the anastomotic site of the LITA-LAD anastomosis is stenotic must be important because it can affect the survival of the patient. Therefore it is imperative to know the quality of the LITA-LAD anastomosis during the operation. 


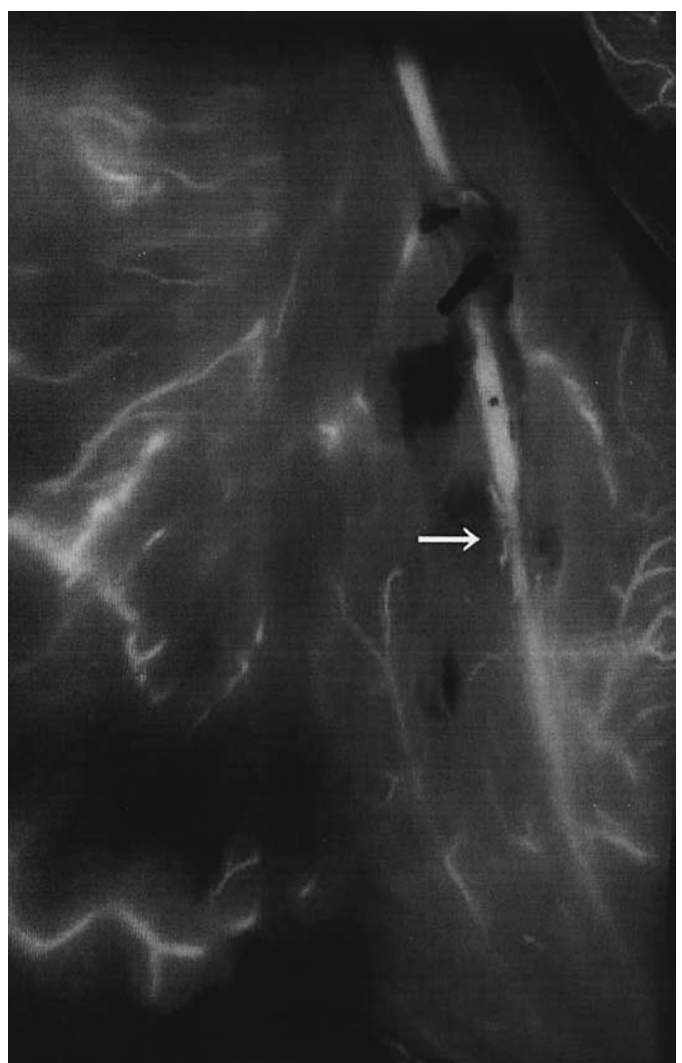

Figure 1. Intraoperative assessment of the LITA-LAD anastomosis with the conventional technique. ICG was injected into the central venous line. Stenosis of the LAD was not so severe in this case, and therefore the LAD and other coronary vessels were enhanced first, followed by the LITA. It was difficult to assess the quality of the anastomosis (arrow).

There are a few reports describing the usefulness of a fluorescence imaging system (the SPY system; Novadaq Technologies, Inc, Mississauga, Ontario, Canada), where ICG was injected through a central venous line. ${ }^{2}$ However, we found that it was difficult to assess the quality of the anastomosis in some cases because native coronary arteries are enhanced first if they are not so narrowed. Actually, we frequently found flow competition between native coronary arteries and grafts, and SPY images were quite different from those obtained with traditional angiography from a practical viewpoint. As we have shown with the images obtained using this new technique, we believe that the images of the SPY system with this technique were equivalent to those of traditional angiography, and it has some advantages, such as being a simple and less-invasive technique and lacking x-ray exposure.

To our knowledge, this is the first report describing direct injection of ICG into the distal ascending aorta to obtain clear images of a LITA-LAD anastomosis intraoperatively in OPCAB. This technique has some limitations, including simultaneous assessment of free grafts directly attached to the proximal ascending aorta. We usually perform 3-dimensional computed tomography in those patients to assess the patency of free grafts.

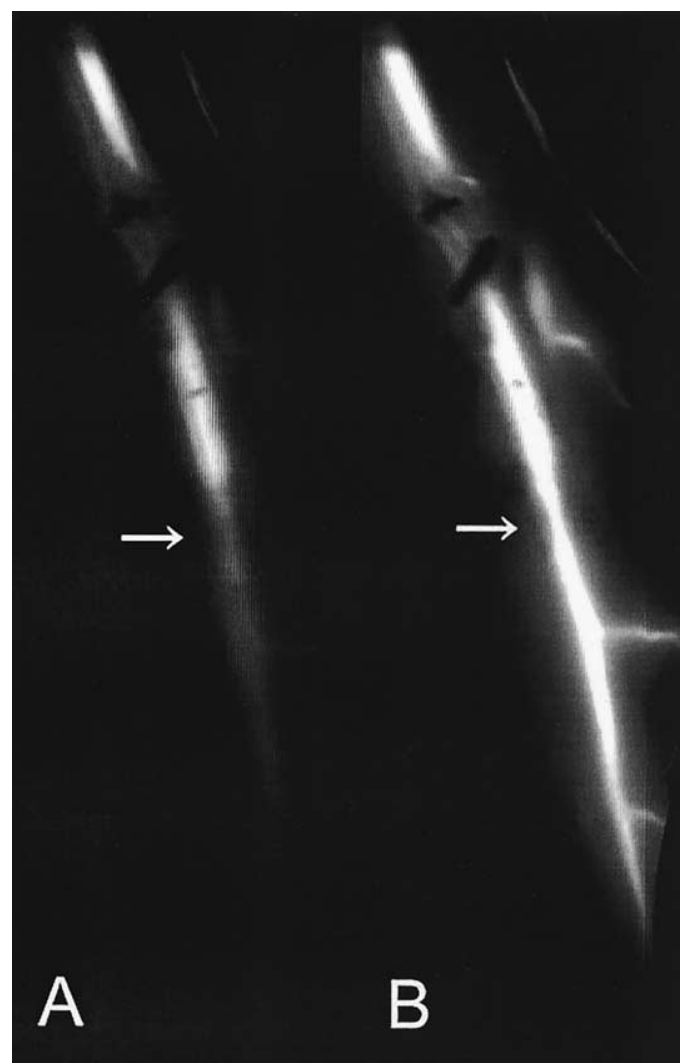

Figure 2. Intraoperative assessment of the LITA-LAD anastomosis with the new technique in the same patient. Two milliliters of ICG was injected directly into the distal ascending aorta. The skeletonized LITA was enhanced first (A), and the LITA and LAD were enhanced subsequently (B). The anastomosis was mildly stenotic (arrow).

\section{References}

1. Racz MJ, Hannan EL, Isom OW, Subramanian VA, Jones RH, Gold JP, et al. A comparison of short- and long-term outcomes after off-pump and on-pump coronary artery bypass graft surgery with sternotomy. J Am Coll Cardiol. 2004;43:557-64.

2. Taggart DP, Choudhary B, Anastasiadis K, Abu-Omar Y, Balacumaraswami L, Pigott DW. Preliminary experience with a novel intraoperative fluorescence imaging technique to evaluate the patency of bypass grafts in total arterial revascularization. Ann Thorac Surg. 2003;75:870-3.

3. Suematsu Y, Takamoto S, Ohtsuka T. Intraoperative echocardiographic imaging of coronary arteries and graft anastomoses during coronary artery bypass grafting without cardiopulmonary bypass. $J$ Thorac Cardiovasc Surg. 2001;122:1147-54.

4. Falk V, Walther T, Kitzinger H, Rauch T, Diegeler A, Autschbach R, et al. An experimental approach to quantitative thermal coronary angiography. Thorac Cardiovasc Surg. 1998;46:25-7.

5. Elbeery JR, Brown PM, Chitwood WR Jr. Intraoperative MIDCABG arteriography via the left radial artery: a comparison with Doppler ultrasound for assessment of graft patency. Ann Thorac Surg. 1998;66: $51-5$. 